Original scientific paper

\title{
A METHOD TO SYNTHESIZE HIGH-PRECISION MOTION CONTROL SYSTEMS FOR UNDERWATER MANIPULATOR
}

\author{
Filaretov, V. F. ${ }^{*, * *}$; Konoplin, A. Y..**; Zuev, A. V. ${ }^{* * * * * * * *} \&$ Krasavin, N. A. ${ }^{* * *}$ \\ ${ }^{*}$ Institute of Automation and Control Processes, Far Eastern Branch of the Russian Academy of \\ Sciences, Vladivostok, 690041, Russia \\ ${ }^{* *}$ Sevastopol State University, Sevastopol, 299053, Russia \\ **** Institute of Marine Technology Problems, Far Eastern Branch of the Russian Academy of Sciences, \\ Vladivostok, 690091, Russia \\ **** Far Eastern Federal University, Vladivostok, 690922, Russia \\ E-Mail: filaretov@inbox.ru, konoplin@marine.febras.ru, zuev@marine.febras.ru, \\ krasavin.na@students.dvfu.ru
}

\begin{abstract}
The development of a method to synthesize combined systems that provide high-precision motion control of multi-joint manipulators mounted on underwater vehicles is presented. This method consists of the following steps. (1) A preliminary analytical calculation of the external torques that occur in all the degrees of mobility of moving underwater manipulators is performed using a recurrent algorithm for solving the inverse dynamics problem. (2) Additional diagnostic observers are then synthesized using the manipulator electric drive's dynamic models and taking into account the analytically estimated external torques. These observers can precisely determine the values of unexpected variations in viscous and coulomb friction torque in all the drives. (3) After that, the precisely identified torque effects on the drives of all the degrees of manipulator's mobility are fully compensated by the adaptive compensator devices that provide stabilization of the dynamic properties of these drives at the nominal level. Results of a numerical simulation showed a significant increase in accuracy of various technological operations performed with an underwater manipulator when the synthesized system was used.

(Received in May 2021, accepted in October 2021. This paper was with the authors 5 weeks for 2 revisions.)
\end{abstract}

Keywords: Underwater Multi-Joint Manipulator, Underwater Vehicle, Identification, HighPrecision, Observer

\section{INTRODUCTION}

Currently, unmanned underwater vehicles (UUV) equipped with multi-joint manipulators (MM) are widely used in a variety of deep-sea operations such as collection of marine fauna and bottom sediment samples, maintenance, repair, and cleaning of underwater technical structures and facilities, including unscrewing of submarine pipeline valves $[1,2]$. Some of these operations are already performed by automatically controlled UUV thanks to the rapid development of technologies and control systems [3-7].

The control accuracy of MM mounted on UUV needs to be further improved in order to extend the range of applications of underwater robotics for performing specific operations $[8,9]$. However, the moving links of underwater MM are affected by significant and difficultto-determine dynamic impacts from the surrounding aquatic environment [10,11]. MM often move underwater objects with unknown mechanical parameters. Besides, during operation of an underwater MM, unexpected increases in viscous and coulomb friction torque may occur in its electric drives (due to variations in the ambient temperature, properties and contamination of the lubricant, or frictions in the sealing connectors depending on the depth of submersion, etc.). As a result, additional variable torques emerge on the output shafts of the MM drives, which significantly reduces the accuracy of motions of the MM working tool (WT) when performing manipulative operations. The challenge of identification of these negative torques 
is the major obstacle in designing an easy-to-implement and high-precision motion control system for underwater MM.

To solve this problem, studies [12-15] proposed some approaches to creating adaptive control systems for underwater MM on the basis of fuzzy logic and sliding modes, which allow the motion accuracy of WT of these MM to be maintained at a desired level taking into account the inaccuracies of the MM models and emerging external disturbances [14]. But the practical application of such systems can be challenging due to difficulties associated with the synthesis of the sliding mode control law. To ensure the required stability margin, robust systems [12] are configured according to the upper limit of acceptable variations in the parameters. And since external effects on the underwater MM drives are hard to assess and they change at a high rate, selecting these parameters is also difficult. A method for assessing the impact of external factors from the viscous water environment on a dynamic model of underwater MM on the basis of neural networks is proposed in [16]. But these networks take a long adjustment time and require the use of powerful (and expensive) computing tools.

For on-line calculation of the force effects of a viscous environment on moving MM links and the joint coupling between their degrees of mobility, algorithms for solving the inverse dynamics problem (IDP) based on various modifications of the recurrent Newton-Euler algorithm can be used. In the works [17, 18], the MM links are represented as uniform cylinders, and the drag force acting on them is calculated by the known equations [10] only for low Reynolds numbers. But this is true only at a low speed of MM motions, when the drag force acting on a link is directly proportional to this speed [19]. When MM links move at high speeds, this relationship becomes quadratic, and, therefore, in studies [20-22] MM links are represented as a set of segments, with the corresponding component of drag force calculated for each segment using the strip theory. The total drag force acting on a link was determined through analytical integration and finding the point of application of this force to each link [23]. Thus, the algorithm for solving the IDP [11] allows calculation of the torque that occurs in each degree of MM mobility under the effect of the drag force on the respective link. This total torque was calculated as a sum of torques created by the drag forces applied to each segment of this link. The modification of the Newton-Euler algorithm used in this work allows taking into account both linear and quadratic relationships of the drag force with the motion speeds of the respective segments of the MM links.

But the analytical calculations using the algorithm for solving IDP are not enough to accurately assess all the negative torque effects on the output shafts of the underwater MM drives. This is due to the great challenge of correctly identifying the parameters of viscous environment's effects on all the MM links, and also determining the values of masses and moments of inertia of the liquid added to these links [23-25]. It is also impossible to analytically estimate unexpected increases in the coulomb and viscous friction torques in the MM electric drives, and also evaluate the influence of underwater objects with unknown parameters moved by these MM.

Values of these negative torques can be specified using the Kalman filter [26] and diagnostic observers [27-30]. But these filters are applicable for linear systems with constant or slowly changing parameters and external effects. Therefore, they cannot be efficiently used to identify rapidly changing external torques in underwater MM drives that are described by complex nonlinear differential equations with significantly and rapidly changing parameters. Unlike filters, the special diagnostic observers allow not only detection of unaccounted torques emerging on the output shafts of the MM drives, but also determination of their values. However, as studies have shown, the accuracy of their measurements is largely determined by the difference between the actual values of the identified functions and those set in the drive models. In underwater MM, the parameters of their drives and external effects can vary within wide ranges (especially when MM moves quickly). Therefore, the parameters and external 
effects that are input in the models of all drives should be as close as possible to the actual ones. The required approximation can be achieved by preliminary calculation of the force effects of the viscous environment on the moving MM links and the joint coupling between their degrees of mobility during a MM motion using the above-mentioned recurrent algorithm [11].

After accurately determining the drive parameters sought and the external torque effects on $\mathrm{MM}$, the well-known approaches can be used [31] to synthesize adaptive compensators that ensure stabilization of the dynamic properties of the underwater MM drives at the nominal level and, thereby, provide the required dynamic control precision. Thus, it is possible to create a hybrid two-step method for synthesizing combined systems that provide high-precision motion control of WT of a MM mounted on an UUV in the aquatic environment.

\section{PROBLEM STATEMENT}

To improve the dynamic precision of the control of MM mounted on an UUV, the present study aims to design a new method for synthesizing combined systems that provide high-precision identification and subsequent compensation for all negative external torques in all degrees of mobility of the underwater MM, which are caused by continuous variations in the parameters of viscous environment's effects on the manipulator in motion. The specified compensation should allow accurate stabilization of the dynamic parameters of all the MM electric actuating drives at the nominal level while maintaining their dynamic properties on the basis of easily implemented control systems.

The main problem set contains two subproblems. The first of them consists of two stages. At the first stage, using the created recurrent algorithm for solving IDP [11], it is suggested to continuously perform an approximate analytical calculation of the torques that occur in all degrees of mobility of MM moving in a viscous aquatic environment, without the effect of the weight of a captured arbitrary object taken into account. At the second stage, it is necessary to accurately identify the unaccounted torque effects by means of the created diagnostic observers. The latter, using the discrepancies formed by them, should not only detect the fact of emergence of additional torques (including possible emerging defects) and accurately determine their values, but also accurately compensate for the consequences of their emergence. The solution of the second subproblem should provide maintenance of accuracy of MM's movements along spatial trajectories (including high-speed ones) in the aquatic environment at a high level by creating adaptive control systems that use the signals and structures generated through solving the first subproblem. A detailed description of the methods and algorithms proposed to solve this problem is given below in the following sections of the work.

\section{CALCULATION OF THE MOMENTS THAT EMERGE IN JOINTS OF MM OPERATED IN THE AQUATIC ENVIRONMENT}

Consider an underwater MM with $n$ degrees of freedom ( $n$-DOF) and an arbitrary kinematic scheme (see Fig. 1), driven by direct-current (DC) motors of independent excitation or with permanent magnets installed at the MM joints. Taking into account the phase coordinates $x_{1}=$ $q, x_{2}=\dot{\alpha}, x_{3}=I$, measured by the respective sensors, dynamics of each electric drive can be described by the following system of equations [29]:

$$
\left\{\begin{array}{c}
\dot{x}_{1}=\frac{1}{i_{p}} x_{2} \\
\dot{x}_{2}=-\frac{k_{v}}{J_{\Sigma}} x_{2}+\frac{k_{m}}{J_{\Sigma}} x_{3}-\frac{M_{c l m b}\left(x_{2}\right)}{J_{\Sigma}}-\frac{P^{*}}{J_{\Sigma}}+d \\
\dot{x}_{3}=-\frac{k_{\omega}}{L} x_{2}-\frac{R}{L} x_{3}+\frac{k_{g}}{L} u
\end{array}\right.
$$


where $q$ is the angle of rotation of the output shaft of the electric drive's gearbox; $\dot{\alpha}$ is the rotation speed of the electric motor rotor; $R, L, I$ are the active resistance, inductance, and current of the armature circuit, respectively; $k_{\omega}, k_{m}, k_{g}$ are the coefficients of counter-EMF, torque, and amplifier gain; $u$ is the input voltage; $J_{\Sigma}$ is the moment of inertia of the motor rotor and the rotating parts of the gearbox, reduced to this rotor; $k_{v}$ is the nominal coefficient of viscous friction; $M_{c l m b}$ is the nominal coulomb friction torque of the motor and its gearbox;

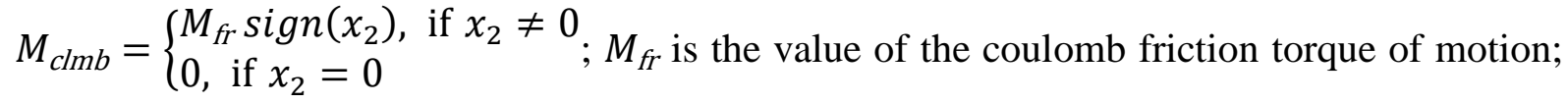
$i_{p}$ is the gearbox ratio; $P^{*}=P / i_{p}$ is the torque $P$ reduced to the motor rotor and caused by joint coupling of underwater MM and viscous environment effects.

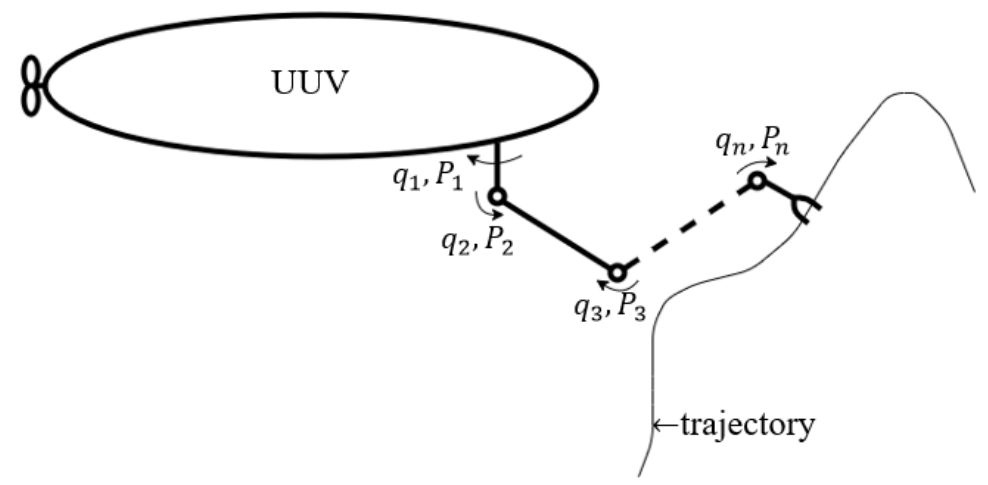

Figure 1: General diagram of an UUV equipped with an $n$-DOF MM.

Since, as noted above, analytical calculation of the $P$ value is very approximate and an unexpected increase in $k_{v}$ and $M_{c l m b}$ values in the electric drives is possible due to the friction in the sealing connectors and the changes in the lubricant properties, the function $d$ is additionally introduced in Eq. (1), which determines the total unknown torque effect described by the Eq. (2):

$$
d=-\frac{1}{J_{\Sigma}}\left[\tilde{k}_{v} x_{2}+\widetilde{M}_{c l m b}\left(x_{2}\right)+\tilde{P}^{*}\right]
$$

where $\tilde{k}_{v}, \widetilde{M}_{c l m b}\left(x_{2}\right)$ are the values of deviations of the viscous friction coefficient and the coulomb friction torque from their nominal values, and $\widetilde{P}^{*}$ is the value of deviation of external torques from the calculated $P^{*}$ value, respectively.

In the first step, a recurrent algorithm for solving the IDP [11] can be used to compute the torques of $P$ acting on the output shafts of all MM drives, which assumes that each elementary segment of the link $i$ of the $n$-DOF MM can have a different speed relative to the fluid at rest not only in magnitude but also in direction. In addition, the force acting on this elementary segment from the viscous fluid can have a linear or quadratic relationship with the speed of this elementary segment in the aquatic environment. Therefore, each link of this $M M$ in the algorithm is divided into a finite number of elementary segments of equal lengths, and the total force acting on the link $i$ is defined as the sum of the forces applied to each elementary segment of this link. As a result, the torques acting in all the degrees of mobility of the underwater MM are calculated using the recurrent formulas starting from the last and ending with the first link [11]:

$$
\begin{aligned}
M_{i} & =A_{i}^{i+1} \cdot M_{i+1}+p_{i}^{*} \times\left(A_{i}^{i+1} \cdot F_{i+1}\right)+r_{i}^{*} \times\left(m_{i} \cdot \ddot{r}_{m i}\right)+r_{p i} \times\left(\Pi_{m i} \cdot \ddot{r}_{m i}\right)+ \\
& +\left(\tau_{i}+T_{i}\right) \cdot \dot{\omega}_{i}+\omega_{i} \times\left(\left(\tau_{i}+T_{i}\right) \cdot \omega_{i}\right)+M_{R p i}+M_{L i}, M_{n+1}=0, i=\overline{n, 1}
\end{aligned}
$$

where $A_{i}^{i+1}$ is the matrix of translation of vectors from $(i+1)^{\text {th }}$ to the $i^{\text {th }}$ coordinate system; $\omega_{i} \in R^{3}$ is the angular velocity of rotation of the link $i ; p_{i}^{*} \in R^{3}$ is the vector coincident with the longitudinal axis of the link $i$, determining the position of the joint $(i+1)$ relative to the 
joint $i ; M_{R p i} \in R^{3}$ is the total torque created by the lateral components of the viscous friction forces acting on each elementary segment of the link $i ; m_{i}$ is the mass of the link $i$; $\dot{\omega}_{i} \in R^{3}$ is the angular acceleration of the link $i ; M_{L i} \in R^{3}$ is the torque created by the component of the angular velocity $\omega_{i}$ directed along the longitudinal axis of the link $i ; \Pi_{m i}$ is the mass of fluid added to the link $i$; $\ddot{r}_{m i} \in R^{3}$ is the linear acceleration of the link $i$ centre of mass; $r_{p i} \in R^{3}$ is the vector indicating the position of the centre of mass $\Pi_{m i}$ relative to the joint $i ; r_{i}^{*} \in R^{3}$ is the vector indicating the centre of mass of the link $i$ relative to the joint $i ; \tau_{i} \in R^{3 \times 3}$ is inertia tensor of the link $i$ relative to its centre of mass; $T_{i} \in R^{3 \times 3}$ is the inertia tensor of the mass of fluid added to the link $i ; F_{i}, M_{i} \in R^{3}$ are the force and the torque of this force acting in the joint $i$, respectively; $(\times)$ and $(\cdot)$ are cross and scalar products of vectors, respectively.

As a result, the calculated external torque $P_{i}$, acting on the output shaft of the $i^{\text {th }}$ electric drive of MM, can be determined by the equality $P_{i}=M_{i(3)}$, where the subscript 3 in parentheses indicates the element's number in the respective vector $M_{i}$. Below, the indices $i$, denoting the number of the corresponding MM degree of mobility, are omitted for simplicity.

\section{DESCRIPTION OF ADDITIONAL OBSERVERS}

As noted above, it is always difficult to accurately assess the force effects from a viscous fluid and, even more so, from objects captured and moved in this environment. As a result, a highquality control of MM based solely on the analytical calculation of the torques $P$ is impossible. In the second step, to determine all the force and torque effects more accurately on all the MM electric drives (to determine values of the functions $d$ ), it is suggested to use special additional diagnostic observers, which can be constructed using the dynamic models of the electric drives of each degree of MM mobility, including external torques $P$ approximately calculated in the first step. Based on the formed discrepancies, these observers are not only to detect the fact of emergence of additional (unaccounted) torques due to the variable interaction of the MM moving a captured object with the viscous fluid, but also to accurately determine the values of these torques, as well as the values of unexpected variations in the torques of viscous and coulomb friction in the electric drives.

While synthesizing diagnostic observers, Eq. (1) is more convenient to be represented in a matrix form:

$$
\left\{\begin{array}{l}
\dot{x}=A x+B u+G\left(x_{2}, P^{*}\right)+D d \\
y=C x
\end{array}\right.
$$

where: $A=\left[\begin{array}{ccc}0 & 1 / i_{p} & 0 \\ 0 & -k_{v} / J_{\Sigma} & k_{m} / J_{\Sigma} \\ 0 & -k_{\omega} / L & -R / L\end{array}\right] ; x=\left[\begin{array}{c}q \\ \dot{\alpha} \\ I\end{array}\right] ; B=\left[\begin{array}{c}0 \\ 0 \\ k_{g} / L\end{array}\right] ; G=\left[\begin{array}{c}0 \\ \frac{-M_{c l m b}\left(x_{2}\right)-P^{*}}{J_{\Sigma}} \\ 0\end{array}\right] ; D=\left[\begin{array}{l}0 \\ 1 \\ 0\end{array}\right] ; C=\left[\begin{array}{l}1 \\ 1 \\ 1\end{array}\right]$.

To identify the value of $d$, we can use diagnostic observers synthesized by the well-known methods described in [29, 30].

The desired observer equation can be described as follows:

$$
\left\{\begin{array}{l}
\dot{x}_{*}=A_{*} x_{*}+B_{*} u+G_{*}+J_{*} y-K e_{y}+D_{*} v \\
y_{*}=C_{*} x_{*}
\end{array}\right.
$$

where $x_{*} \in R^{k}$ is the vector of observer's state; $y_{*}$ is the output signal; $A_{*} \in R^{k \times k}$ is the matrix of observer's dynamic properties; $B_{*} \in R^{k}$ is the vector under control of $u$; $G_{*} \in R^{k}$ is the vector of external disturbances; $J_{*} \in R^{k \times m}$ is the matrix taking into account the measurable variables of the system's state in the observer; $K$ is the positive constant; $k$ is the observer's order; $m$ is the number of sensors that measure the system's state variables; $C_{*} \in R^{k}$ is the row vector; $e_{y}=\Phi x-x_{*} \in R^{3}$ is the discrepancy that allows determination of differences between the actual parameters of the respective MM electric drives and their nominal parameters in the 
system (4) (if $e_{y} \neq 0$ and $d \neq 0$ ); $\Phi \in R^{3}$ is the vector to be determined; the function $v$ has the form $v=\left\{\begin{array}{c}-g_{*} \frac{e_{y}}{\left|e_{y}\right|}, \text { if } e_{y} \neq 0 \\ 0, \text { else }\end{array}, g_{*}\right.$ is the positive constant.

When synthesizing the observer that determines the emergence of a non-zero function $d$ in the system (4), the matrices $A_{*}, B_{*}$ and vectors $J_{*}, \Phi$ should be defined.

The canonical forms of the matrix $A_{*}$ and vector $C_{*}$ are as follows:

$$
A_{*}=\left[\begin{array}{ccccc}
0 & 1 & 0 & \ldots & 0 \\
0 & 0 & 1 & \cdots & 0 \\
& \vdots & & \ddots & \vdots \\
0 & 0 & 0 & \cdots & 0
\end{array}\right], \quad C_{*}=\left[\begin{array}{cccc}
1 & 0 & \ldots & 0
\end{array}\right]
$$

If there are no defects, the equalities:

$$
\begin{aligned}
& x_{*}=\Phi x \\
& y_{*}=R_{*} y
\end{aligned}
$$

are fulfilled that satisfy the conditions:

$$
\begin{gathered}
\Phi A=A_{*} \Phi+J_{*} C \\
R_{*} C_{*}=C \Phi
\end{gathered}
$$

from which expressions can be derived for the rows of matrices $\Phi$ and $J_{*}$ :

$$
\begin{gathered}
\Phi_{1}=R_{*} C \\
\Phi_{i} A=\Phi_{i+1}+J_{* i} A, i=\overline{1, k-1} \\
\Phi_{k} A=J_{* k} A
\end{gathered}
$$

where $\Phi_{i}$ and $J_{* i}$ are the $i^{\text {th }}$ rows of the matrices $\Phi$ and $J_{*}, i=1, \ldots, k$.

By introducing the known condition [30] $\Phi D \neq 0$ of the sensitivity or discrepancy in $d$, the vector $\Phi$ can be formulated as $\Phi=\left[\begin{array}{lll}0 & 1 & 0\end{array}\right]$.

It is advisable to solve the problem of constructing diagnostic observers in the canonical Kronecker's form [30]. In this case, taking into account the dimension of the synthesized observers, the equalities $A_{*}=0, C_{*}=1$ are valid. With this equality taken into account and the validity of the relationships [30]:

$$
\begin{aligned}
& J_{*}=\Phi A \\
& B_{*}=\Phi B \\
& G_{*}=\Phi G \\
& D_{*}=\Phi D
\end{aligned}
$$

the desired observer can be described by the following equation:

where

$$
\dot{x}_{*}=-\frac{k_{v}}{J_{\Sigma}} \dot{\alpha}+\frac{k_{m}}{J_{\Sigma}} I-\frac{M_{c l m b}\left(x_{2}\right)}{J_{\Sigma}}-\frac{P^{*}}{J_{\Sigma}}-K e_{y}+v
$$

$$
e_{y}=\dot{\alpha}-x_{*}
$$

It is known [29] that when $g_{*}>|d|$ is selected, the estimation error is $e_{y} \rightarrow 0$ with $t \rightarrow \infty$. Meeting this condition provides stability of the synthesized observer, which can be proved by studying the Lyapunov's function [28]. In this case, the value of $d$ can be estimated with high accuracy using a low-pass filter [32]: $\hat{d}=v_{f}$, where $\hat{d}$ is the estimate of the function $d$ obtained by the observer (17), and $v_{f}$ is the value of the function $v$ passed through the low-pass filter.

Thus, the use of additional observers (17) for electric drives of each degree of mobility of MM provides detection and evaluation of unaccounted total torques $\hat{d}$ that determine the $\tilde{P}^{*}$ value of deviations of the torques from the previously and approximately calculated $P^{*}$ values, 
as well as deviations of the viscous and coulomb friction torques in these drives from their nominal values.

Thus, the use of the algorithm for preliminary analytical calculation of IDP in the first step of the method designed in the present article makes it possible to significantly simplify the problem of identifying external effects and bring their values closer to the actual ones.

\section{SYNTHESIS OF ADAPTIVE COMPENSATOR DEVICES}

To accurately compensate for all identified external torques in all degrees of mobility of MM and stabilize the parameters of all its electric drives on the nominal level, it is necessary to synthesize adaptive compensator devices (ACD) that would provide the required accuracy of operation of these drives, even with rapid variations in external torques, and also with varying torques of coulomb and viscous friction in these drives. These ACD are suggested to be synthesized using an approach that consists in stabilization of parameters of the differential equations describing the operation of the MM drives [31,33], which would provide invariant operation of these drives with all load variations. For this, the differential Eq. (1) should be revised for each loaded electric drive, so that the equality $\dot{M}_{c l m b}=0$ is valid for the drives on move:

$$
u k_{g} k_{m}=L J_{\Sigma} \ddot{\alpha}+\left(k_{v} L+J_{\Sigma} R\right) \ddot{\alpha}+\left(R k_{v}+k_{\omega} k_{m}\right) \dot{\alpha}+L\left(\dot{P}^{*}+\dot{\hat{d}}\right)+R\left(P^{*}+\hat{d}+M_{c l m b}\right)
$$

The desired linear differential equation for each electric drive with nominal constant parameters and stable dynamic properties has the following form:

$$
\hat{u} k_{g} k_{m}=L J_{\Sigma} \ddot{\alpha}+J_{\Sigma} R \ddot{\alpha}+k_{\omega} k_{m} \dot{\alpha}
$$

where $\hat{u}$ is the input control signal of ACD.

By expressing the value of the highest derivative $\dddot{\alpha}$ from Eq. (20)

$$
\dddot{\alpha}=\frac{\hat{u} k_{g} k_{m}-J_{\Sigma} R \ddot{\alpha}-k_{\omega} k_{m} \dot{\alpha}}{L J_{\Sigma}}
$$

and substituting it into the original Eq. (19), it is easy to obtain the desired control law that would provide the desired stable quality of operation of each MM electric drive:

$$
u=\frac{1}{k_{g} k_{m}}\left[k_{v} L \ddot{\alpha}+k_{v} R \dot{\alpha}+L\left(\dot{P}^{*}+\dot{\hat{d}}\right)+R\left(P^{*}+\hat{d}+M_{c l m b}\right)\right]+\hat{u}
$$

In modern MM electric drives, the electrical time constant is usually negligible $\left(\frac{L}{R} \ll 1\right)$, and, therefore, this Eq. (22) will have the following form:

$$
u=\frac{1}{k_{g} k_{m}}\left[k_{v} R \dot{\alpha}+R\left(P^{*}+\hat{d}\right)+M_{c l m b} R\right]+\hat{u}
$$

To impart the desired quality characteristics and dynamic properties to the electric drive described by Eq. (20) with constant parameters, a typical additional compensator device (TACD) can be installed in its direct circuit. A general diagram of the electric drive that controls the $i^{\text {th }}$ degree of mobility of the underwater MM is shown in Fig. 2. In this figure, new designations are introduced: PS, the position sensor of the output shaft of the electric drive's gearbox; SS, the rotor rotation speed sensor; DO, the diagnostic observer described by Eq. (17); BPC, the block for preliminary calculation of external torques $P^{*} ; q^{*}$, the input (desired) signal.

Implementation of the electric drive shown in Fig. 2 through precise stabilization of the parameters of the drives' differential equations at the nominal level ensures accurate operation of the entire MM, even if a simple TACD is used. 


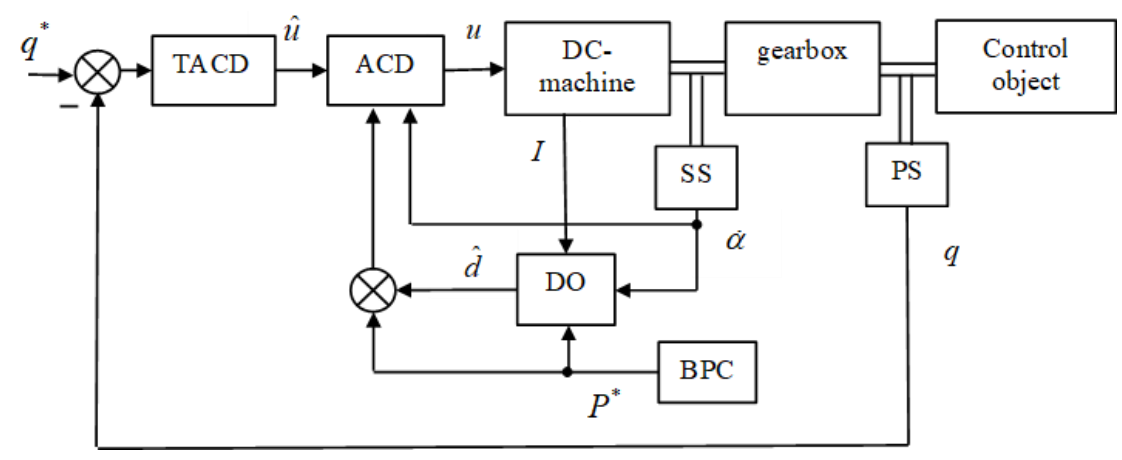

Figure 2: Diagram of an electric drive of underwater MM.

\section{SIMULATION RESULTS}

To study the accuracy of system operation (see Fig. 2), a motion of a 3-DOF MM with a PUMA kinematic scheme [11,34], which has only three translational degrees of mobility, was simulated in Matlab Simulink. Its WT moved along a trajectory (see Fig. 3) described by the following system of equations [35]:

$$
\left\{\begin{array}{c}
\dot{x}^{*}=\frac{V^{*}}{\sqrt{\left(\frac{\partial f_{y}\left(x^{*}\right)}{\partial x^{*}}\right)^{2}+\left(\frac{\partial f_{z}\left(x^{*}\right)}{\partial x^{*}}\right)^{2}+1}} \\
y^{*}=f_{y}\left(x^{*}\right) \\
z^{*}=f_{z}\left(x^{*}\right)
\end{array}\right.
$$

where $V^{*}=0.6 \mathrm{~m} / \mathrm{s}$ is the modulus of the vector of the desired speed of WT motion along the specified spatial trajectory; $f_{y}\left(x^{*}\right)=0.5 \sin (10 x)+0.05 ; f_{z}\left(x^{*}\right)=0.01$. The trajectory motion time is $4 \mathrm{~s}$.

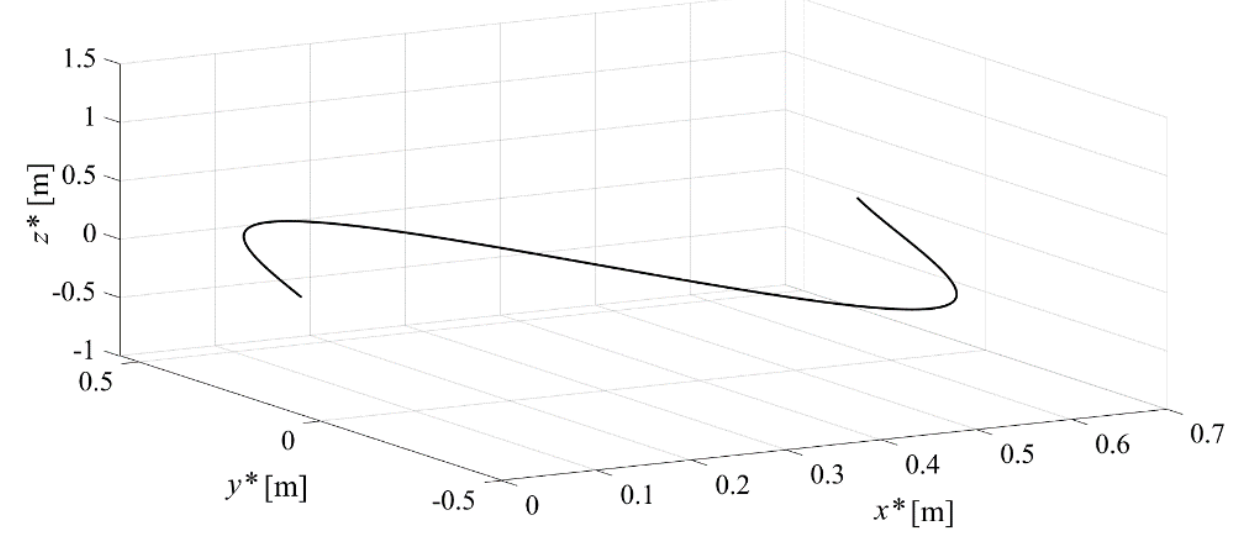

Figure 3: Trajectory of the MM WT motion.

For simulation, the following MM parameters were used [11, 34]: $l_{1}=0.05 \mathrm{~m} ; l_{2}=l_{3}=$ $0.5 \mathrm{~m} ; m_{1}=0.4 \mathrm{~kg} ; m_{2}=m_{3}=3.925 \mathrm{~kg} ;\left|r_{1}^{*}\right|=0.025 \mathrm{~m},\left|r_{2}^{*}\right|=\left|r_{3}^{*}\right|=0.25 \mathrm{~m}$. It was assumed that all centres of mass of the links coincide with the centres of buoyancy; the links are cylindrical in shape with a base radius of $r_{1}=r_{2}=r_{3}=0.05 \mathrm{~m}$ and neutral buoyancy. Their inertia tensors are diagonal and have the following values: $\tau_{1_{11}}=6 \times 10^{-4} \mathrm{kgm}^{2}$, $\tau_{1_{22}}=\tau_{1_{33}}=4 \times 10^{-4} \mathrm{kgm}^{2}, \tau_{2_{11}}=\tau_{3_{11}}=5 \times 10^{-3} \mathrm{kgm}^{2}, \tau_{2_{22}}=\tau_{2_{33}}=\tau_{3_{22}}=\tau_{3_{33}}=$ $8.4 \times 10^{-2} \mathrm{kgm}^{2}$. The drag coefficients used in the algorithm of solving the IDP [11], as well as the added masses of fluid of each MM link, have the following values: $k_{1}^{*}=k_{2}^{*}=k_{3}^{*}=0.6$, $k_{L 1}=k_{L 2}=k_{L 3}=0.12 ; \quad \Pi_{1}=0.1 \mathrm{~kg} ; \Pi_{2}=\Pi_{3}=1.075 \mathrm{~kg}$. The water density is 
$1000 \mathrm{~kg} / \mathrm{m}^{3}$. The diagonal elements of the corresponding inertia tensors of the added liquid masses are: $T_{1_{11}}=1 \times 10^{-4} \mathrm{kgm}^{2}, T_{1_{22}}=T_{1_{33}}=1.5 \times 10^{-4} \mathrm{kgm}^{2}, T_{2_{11}}=T_{3_{11}}=1.4 \times$ $10^{-3} \mathrm{kgm}^{2}, T_{2_{22}}=T_{2_{33}}=T_{3_{22}}=T_{3_{33}}=0.026 \mathrm{kgm}^{2}$. It was assumed that the equivalent electric drives with DC motors of independent excitation or permanent magnets, installed at all degrees of mobility of MM, have the following parameters: $J_{\Sigma}=1 \times 10^{-4} \mathrm{kgm}^{2}, R=0.2 \mathrm{Ohm}$, $k_{g}=35, i_{p}=100, k_{\omega}=0.02 \mathrm{Vs}, k_{\omega}=0.02 \mathrm{Vs}, k_{m}=0.02 \mathrm{Nm} / \mathrm{A}, k_{v}=0.005 \mathrm{Nms} / \mathrm{rad}$, $M_{f r}=0.06 \mathrm{Nm}, L=0.002 \mathrm{H}$. The observers (17) with parameters $k=10$ and $g_{*}=7000$ were used for each electric drive. In all the electric drives, ACD (23) and TACD (see Fig. 2) were used. In this case, TACD are proportional-integral-derivative controllers with the following coefficients: $k_{P}=10, k_{I}=0.01, k_{D}=0.4$.

To simulate additional external torques $\tilde{P}^{*}$ in the algorithm for solving IDP, the used values of viscous friction coefficients, added masses, and moments of inertia of liquid were increased by $20 \%$. In addition, at time $t=2 \mathrm{~s}$, in MM electric drives of all degrees of mobility, the coefficients of viscous friction and coulomb friction torques were also by $20 \%$ higher than their nominal values.

Fig. 4 shows the patterns of variations in dynamic errors $\varepsilon$ of the WT motion along certain programmed trajectory (see Fig. 3): without the synthesized ACD in MM electric drives (black curve); with the use of ACD (23) (green curve) that take into account only the calculated $P^{*}$ values; the red curve shows the accuracy of the system with additional observers used (17). As can be seen in Fig. 3, the $\varepsilon$ value reaches $32 \mathrm{~mm}$ with no ACD and does not exceed $15 \mathrm{~mm}$ with ACD used. The introduction of additional observers, which allows taking into account values of $\tilde{P}^{*}, \tilde{k}_{v}$, and $\widetilde{M}_{c l m b}\left(x_{2}\right)$, leads to a decrease in the dynamic error of the MM WT motion to $4 \mathrm{~mm}$ in most segments.

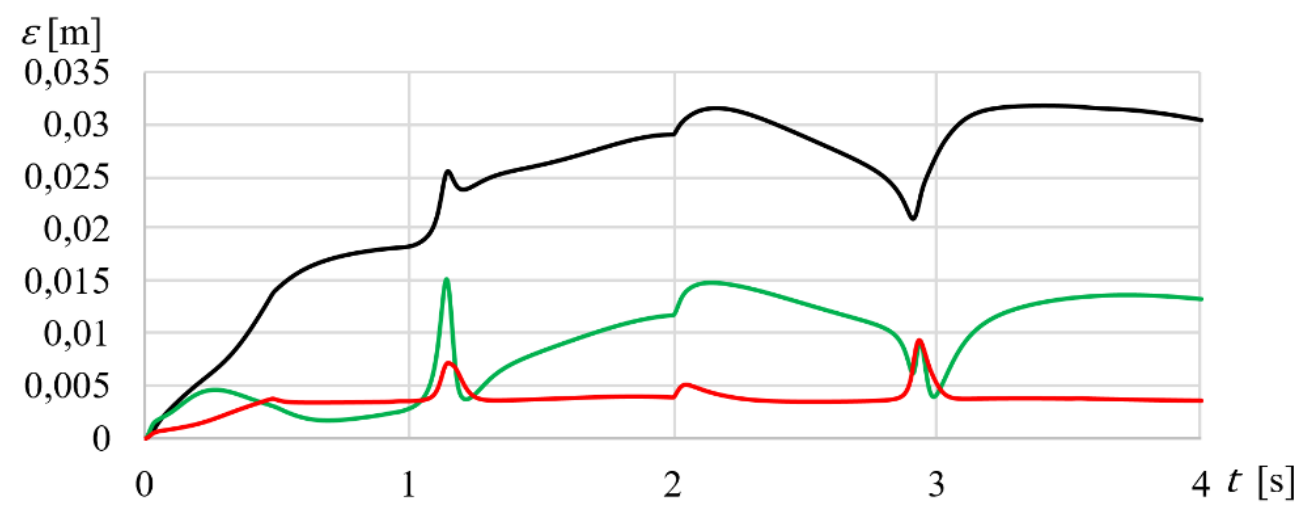

Figure 4: Values of dynamic errors of the MM WT motion along a sinusoidal trajectory.

Also, a simulation was carried out for a MM WT motion with a constant speed of $V^{*}=$ $0.45 \mathrm{~m} / \mathrm{s}$ along a circular trajectory described by a system of equations (see Fig. 5):

$$
\left\{\begin{array}{c}
x^{*}=0.3 \sin (1.5 t)+0.4 \\
y^{*}=0.3 \cos (1.5 t)+0.4 \\
z^{*}=0.01
\end{array}\right.
$$

Fig. 5 shows that after the completion of transients processes, the dynamic error of the WT motion with no ACD used in its drives reaches $24 \mathrm{~mm}$ (black curve); when ACD are used (green curve) with only calculated $P^{*}$ values taken into account, the $\varepsilon$ value does not exceed $12 \mathrm{~mm}$. With additional observers introduced, the dynamic error decreases to $3 \mathrm{~mm}$ (red curve).

Thus, the results of numerical simulation confirm the efficiency of the synthesized system which allows a significant increase in the accuracy of various technological operations performed with the WT of an underwater MM. 


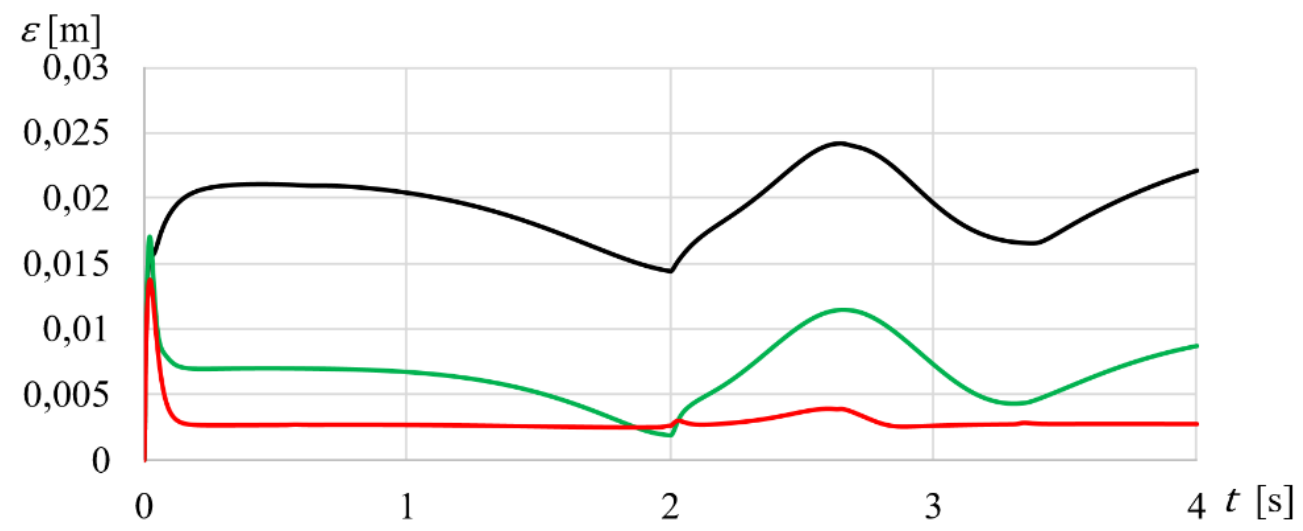

Figure 5: Values of dynamic errors of the MM WT motion along a circular trajectory.

\section{CONCLUSION}

The article describes a new method to synthesize combined systems of high-precision motion control for operating the WT of an underwater MM. These systems provide high-precision identification and subsequent compensation for negative effects of variable external torques in the respective degrees of mobility of MM that occur during arbitrary spatial motion of their links in a viscous aquatic environment. Accurate compensation for these effects allows stabilizing the parameters of all actuating electric drives at the nominal level and, thereby, maintaining the required dynamic control precision.

At the first stage of the synthesis, an analytical calculation of the moments emerging in all degrees of mobility of MM during their motion in a viscous aquatic environment is carried out using a recurrent algorithm for solving IDP. Since this calculation is approximate due to the impossibility to accurately determine the parameters of interaction of all the MM links and the captured objects with the aquatic environment, it is suggested to build additional diagnostic observers using the dynamic models of electric drives of each degree of MM mobility, including approximately calculated external torques. These observers, based on the discrepancies formed by them, not only detect the fact of additional (unaccounted) torques emerging due to the interaction of the moving MM with the viscous fluid, but also accurately determine their values, as well as the values of unexpected variations in the torques of viscous and coulomb friction in the electric drives.

To accurately compensate for all identified external torques' effects on all the MM electric drives, it is suggested to use ACD which allow maintaining a high accuracy of motions of underwater MM along any spatial trajectories at an arbitrary speed. A simulation of operation of the synthesized systems with additional observers showed a multifold reduction in errors of MM WT motion along a complex spatial trajectory vs. the use of typical control systems. This confirms the operability and high efficiency of the proposed method.

To ensure the required accuracy of the system synthesized on the basis of the method proposed in the article, it is important to choose the appropriate MM electric drives power, since when drives enter saturation mode (with insufficient power), the MM WT can deviate significantly and unpredictably from desired trajectories especially in areas with a large curvature. This can lead to emergency situations since the MM in these areas lose control.

Further research of the authors will focus on the creation of methods for the formation of high-speed operation modes of the underwater MM installed on the UUV. These methods will make it possible to form variable velocities of the MM along complex spatial trajectories, at which there will be no significant displacements of the UUV from the stabilization points and significant deviations of the MM WT from the objects of the performed operations. 


\section{ACKNOWLEDGEMENTS}

The work was financially supported by the Russian Foundation for Basic Research (RFBR), grants nos. 20-38-70161 and 19-08-00347_A, and by Sevastopol State University within the framework of an internal grant to attract leading researchers (project identifier 42-01-09/169/2021-5).

\section{REFERENCES}

[1] Bae, J.; Bak, J.; Jin, S.; Seo, T.; Kim, J. (2018). Optimal configuration and parametric design of an underwater vehicle manipulator system for a valve task, Mechanism and Machine Theory, Vol. 123, 76-88, doi:10.1016/j.mechmachtheory.2018.01.014

[2] Sivčev, S.; Coleman, J.; Omerdić, E.; Dooly, G.; Toal, D. (2018). Underwater manipulators: A review, Ocean Engineering, Vol. 163, 431-450, doi:10.1016/j.oceaneng.2018.06.018

[3] Marani, G.; Yuh, J. (2014). The SAUVIM underwater vehicle-manipulator system, Marani, G.; Yuh, J. (Eds.), Introduction to Autonomous Manipulation, Springer, Berlin, 79-105, doi:10.1007/978-3-642-54613-6_4

[4] Johansson, B.; Siesjö, J.; Furuholmen, M. (2010). Seaeye Sabertooth: A hybrid AUV/ROV offshore system, Proceedings of the OCEANS 2010 IEEE/MTS, 3 pages, doi:10.1109/ OCEANS.2010.5663842

[5] Lane, D. M.; Maurelli, F.; Kormushev, P.; Carreras, M.; Fox, M.; Kyriakopoulos, K. (2012). Persistent autonomy: the challenges of the PANDORA project, IFAC Proceedings Volumes, Vol. 45, No. 27, 268-273, doi:10.3182/20120919-3-IT-2046.00046

[6] Filaretov, V. F.; Konoplin, A. Y.; Konoplin, N. Y. (2019). System for automatic soil sampling by AUV equipped with multilink manipulator, International Journal of Energy Technology and Policy, Vol. 15, No. 2/3, 208-223, doi:10.1504/ijetp.2019.098965

[7] Marani, G.; Choi, S. K.; Yuh, J. (2009). Underwater autonomous manipulation for intervention missions AUVs, Ocean Engineering, Vol. 36, No. 1, 15-23, doi:10.1016/j.oceaneng.2008.08.007

[8] Konoplin, A. Y.; Konoplin, N. Y.; Shuvalov, B. V. (2019). Technology for implementation of manipulation operations with different underwater objects by AUV, Proceedings of the 2019 International Conference on Industrial Engineering, Applications and Manufacturing, 5 pages, doi:10.1109/ICIEAM.2019.8743094

[9] Conti, R.; Meli, E.; Ridolfi, A.; Allotta, B. (2015). An innovative decentralized strategy for I-AUVs cooperative manipulation tasks, Robotics and Autonomous Systems, Vol. 72, 261-276, doi:10.1016/j.robot.2015.06.006

[10] Coiffet, P. (1983). Interaction with the Environment: Robots Sensors and Sensing, $2^{\text {nd }}$ edition, Kluwer Academic Publishers, Amsterdam

[11] Filaretov, V. F.; Konoplin, A. Y. (2015). System of automatic stabilization of underwater vehicle in hang mode with working multilink manipulator, Proceedings of the 2015 International Conference on Computer, Control, Informatics and its Applications, 132-137, doi:10.1109/ IC3INA.2015.7377760

[12] Londhe, P. S.; Mohan, S.; Patre, B. M.; Waghmare, L. M. (2017). Robust task-space control of an autonomous underwater vehicle-manipulator system by PID-like fuzzy control scheme with disturbance estimator, Ocean Engineering, Vol. 139, 1-13, doi:10.1016/j.oceaneng.2017.04.030

[13] Zhou, Z.; Tang, G.; Huang, H.; Han, L.; Xu, R. (2020). Adaptive nonsingular fast terminal sliding mode control for underwater manipulator robotics with asymmetric saturation actuators, Control Theory and Technology, Vol. 18, No. 1, 81-91, doi:10.1007/s11768-020-9127-0

[14] Yang, C.; Yao, F.; Zhang, M.; Zhang, Z.; Wu, Z.; Dan, P. (2020). Adaptive sliding mode PID control for underwater manipulator based on Legendre polynomial function approximation and its experimental evaluation, Applied Sciences, Vol. 10, No. 5, Paper 1728, 15 pages, doi:10.3390/app10051728

[15] Zhong, Y.; Yang, F. (2019). Dynamic modeling and adaptive fuzzy sliding mode control for multilink underwater manipulators, Ocean Engineering, Vol. 187, Paper 106202, 11 pages, doi: $\underline{10.1016 / \text { j.oceaneng.2019.106202 }}$ 
[16] Salloom, T.; Yu, X.; He, W.; Kaynak, O. (2020). Adaptive neural network control of underwater robotic manipulators tuned by a genetic algorithm, Journal of Intelligent \& Robotic Systems, Vol. 97, No. 3, 657-672, doi:10.1007/s10846-019-01008-y

[17] Craig, J. J. (2005). Manipulator dynamics, Craig, J. J. (Ed.), Introduction to Robotics: Mechanics and Control, Pearson Education International, New Jersey, 165-200

[18] Wang, T.; You, Z.; Song, W.; Zhu, S. (2020). Dynamic analysis of an underwater cable-driven manipulator with a fluid-power buoyancy regulation system, Micromachines, Vol. 11, No. 12, Paper 1042, 14 pages, doi: $10.3390 / \mathrm{mi} 11121042$

[19] Koval, E. V. (1994). Automatic stabilization system of underwater manipulation robot, Proceedings of OCEANS'94, 807-812, doi:10.1109/oceans.1994.363832

[20] Tarn, T. J.; Shoults, G. A.; Yang, S. P. (1996). A dynamic model of an underwater vehicle with a robotic manipulator using Kane's method, Autonomous Robots, Vol. 3, No. 2, 269-283, doi:10.1007/bf00141159

[21] Lévesque, B.; Richard, M. J. (1994). Dynamic analysis of a manipulator in a fluid environment, The International Journal of Robotics Research, Vol. 13, No. 3, 221-231, doi:10.1177/ $\underline{027836499401300304}$

[22] McLain, T. W.; Rock, S. M.; Lee, M. J. (1996). Experiments in the coordinated control of an underwater arm/vehicle system, Yuh, J.; Ura, T.; Bekey, G. A. (Eds.), Underwater Robots, Springer, Boston, 139-158, doi:10.1007/978-1-4613-1419-6 8

[23] Sharma, A. K.; Saha, S. K. (2021). Simplified drag modeling for the dynamics of an underwater manipulator, IEEE Journal of Oceanic Engineering, Vol. 46, No. 1, 40-55, doi:10.1109/joe.2019.2948412

[24] Lin, C.-C.; Chen, R.-C.; Li, T.-L. (1999). Experimental determination of the hydrodynamic coefficients of an underwater manipulator, Journal of Robotic Systems, Vol. 16, No. 6, 329-338, doi:10.1002/(sici)1097-4563(199906)16:6<329::aid-rob2>3.0.co;2-5

[25] McLain, T. W.; Rock, S. M. (1998). Development and experimental validation of an underwater manipulator hydrodynamic model, The International Journal of Robotics Research, Vol. 17, No. 7, 748-759, doi:10.1177/027836499801700705

[26] Zhang, Q. (2018). Adaptive Kalman filter for actuator fault diagnosis, Automatica, Vol. 93, 333342, doi:10.1016/j.automatica.2018.03.075

[27] He, J.; Zhang, C. (2012). Fault reconstruction based on sliding mode observer for nonlinear systems, Mathematical Problems in Engineering, Vol. 2012, Paper 451843, 22 pages, doi:10.1155/2012/451843

[28] Zhirabok, A.; Zuev, A.; Shumsky, A. (2020). Fault identification for systems described by nonlinear models using sliding mode observers, Proceedings of the $15^{\text {th }}$ IEEE Conference on Industrial Electronics and Applications, 314-319, doi:10.1109/ICIEA48937.2020.9248220

[29] Zhirabok, A.; Zuev, A.; Shumsky, A. (2019). Sliding mode observers based fault identification in mechatronic systems, Proceedings of the $45^{\text {th }}$ Annual Conference of the IEEE Industrial Electronics Society, 161-166, doi:10.1109/IECON.2019.8926898

[30] Zhirabok, A. N.; Shumsky, A. E.; Pavlov, S. V. (2017). Diagnosis of linear dynamic systems by the nonparametric method, Automation and Remote Control, Vol. 78, No. 7, 1173-1188, doi: $10.1134 / \mathrm{s} 0005117917070013$

[31] Filaretov, V. (2007). Synthesis of adaptive control systems for electric servo actuators of manipulators, Proceedings of the $18^{\text {th }}$ DAAAM International Symposium, 277-278

[32] Utkin, V. I. (1992). Sliding Modes in Control Optimization, Springer-Verlag, Berlin

[33] Filaretov, V. F.; Zhirabok, A. N.; Zuev, A. V.; Protcenko, A. (2012). The development of the faults accommodation system for actuators of multilink manipulators, Proceedings of the $23^{\text {rd }}$ DAAAM International Symposium, 575-578

[34] Filaretov, V. F.; Konoplin, A. Y. (2015). System of automatically correction of program trajectory of motion of multilink manipulator installed on underwater vehicle, Procedia Engineering, Vol. 100, 1441-1449, doi:10.1016/j.proeng.2015.01.514

[35] Filaretov, V. F.; Yukhimets, D. A.; Konoplin, A. Y. (2014). Synthesis of system for automatic formation of multilink manipulator velocity, Proceedings of the $2^{\text {nd }}$ RSI/ISM International Conference on Robotics and Mechatronics, 785-790, doi:10.1109/ICRoM.2014.6990999 